\title{
Phytotherapie bei Verbrennungen und Sonnenbrand
}

Sonnenbrand ist meist eine Verbrennung I.-II. Grades Verschiedene Phytotherapeutika eignen sich für die Behandlung Petra Staubach

\section{Zusammenfassung}

Sonnenbrände sind die häufigste Ursache für Verbrennungen. Da diese das Auftreten von Hauttumoren begünstigen, ist eine adäquate Prophylaxe wichtig. Zur Behandlung von Verbrennungen I. und II. Grades eignen sich Phytotherapeutika. Der Beitrag gibt eine Übersicht über die verschiedenen Möglichkeiten. 
Verbrennungen kommen im Alltag häufig vor.

Ursachen dafür sind [11]:

- Ausgeprägte Exposition mit Wärme (z. B. Sonnenbrand,

kochendes Wasser)

- Kältequelle

- Intensive Reibung

- Kontakt mit chemischen Substanzen

- Exposition mit radioaktiver Elemente (Strahlen) oder

- Kontakt mit Elektrizität (Strom, Blitz)

Häufigste Ursache sind Verbrennungen nach ausgeprägten Sonnenbaden (Sonnenbränden), die meist nur mild sind und mit einem diskreten Erythem und einem Spannungsgefühl der Haut einhergehen. Dies ist laut Definition bereits eine Verbrennung I. Grades. Bekannt ist, dass rezidivierende Sonnenbrände (beginnend im Kindesalter) zum vermehrten Auftreten von Hauttumoren, z. B. Melanomen, Basaliomen und Spinaliomen, führen. Die Patienten müssen über diese Risiken aufgeklärt werden und eine adäquate Beratung zum Sonnenschutz erhalten.

\section{Einteilung der Verbrennungen}

Je nach Dauer und Intensität der Schädigung, unabhängig von dem Ausmaß (Tab. 1), werden verschiedene Grade unterschieden [11]:

\section{Verbrennung I. Grades (Combustio erythematosa)}

Hier ist de obere Epidermis betroffen. Klinisch imponieren Erythem, Ödem begleitet von einem brennenden Schmerz.

\section{Verbrennung II. Grades (Combustio bullosa)}

Hier ist die Schädigung der Haut ausgeprägter, ergänzend entwickelt sich subepidermal eine Blase. Durch die Zerstörung der Blase kommt es zur Verkrustung begleitet von starken Schmerzen.

\section{Verbrennung III. Grades (Combustio necroticans)}

Die Epidermis ist zerstört, auch die Hautanhangsgebilde in der Dermis sind betroffen bis zur Muskulatur, dem Fettgewebe usw. Es bilden sich Nekrosen, Abheilung unter Narbenbildung, ggf Analgesie.

\section{Verbrennung IV. Grades (Verkohlung)}

Zerstörung aller Hautschichten und tieferer Strukturen, Nekrosen, Narbenbildung, Analgesie.

\section{Therapie Allgemein [11]}

Schnelles Handeln ist bei Verbrennungen allgemein erforderlich. Grundsätzlich sollte die Haut mit fließendem kaltem Wasser ( 15C) benetzt werden oder sonstige Kälteexposition (nicht zu kalt, bei Eis-

\section{Wichtige Ansprechpartner}

Bei Fragen steht hier bundesweit und auch international die Giftzentrale der Universitätsmedizin Mainz unter

Tel. $+496131 / 19240$ oder $+496131 / 232466$, Fax $+496131 / 176605$ oder $+496131 / 232468$ www.giftinfo.uni-mainz.de zur Verfügung.

\begin{tabular}{|c|c|c|c|}
\hline \multicolumn{4}{|c|}{ Ausmaß der Verbrennung: Neunerregel nach Wallace } \\
\hline Körperteil & 0-1 Jahr & Kind & Erwachsener \\
\hline Kopf & $20 \%$ & $16 \%$ & $9 \%$ \\
\hline Rumpf & $30 \%$ & $32 \%$ & $36 \%$ (2 mal 18) \\
\hline Arme & $18 \%$ & $18 \%$ & $18 \%$ (2 mal 9) \\
\hline Hand & $1 \%$ & $1 \%$ & $1 \%$ (nur 1-mal) \\
\hline Genitalregion & $1 \%$ & $1 \%$ & $1 \%$ (nur $1 \mathrm{mal}$ ) \\
\hline Oberschenkel & $15 \%$ & $16 \%$ & $18 \%$ (2 mal 9) \\
\hline Unterschenkel/Fuß & $15 \%$ & $16 \%$ & $18 \%$ (2 mal 9) \\
\hline
\end{tabular}

Tab. 1

packs Unterlage verwenden), feuchte Umschläge über einen Zeitraum von mind. 10 min - je nach Intensität des Schmerzes.

Sobald die Wunden erosiv sind oder auch größere Flächen betroffen sind, müssen zusätzlich antiseptische Externa zur Anwendung kommen und je nach Ausmaß kann auch eine antibiotische Systemtherapie erforderlich sein.

Mögliche chemische Auslöser der Verbrennungen, z. B. Intoxikationen, sollten vor Therapie in Erfahrung gebracht werden, um eine spezifische Therapie einzuleiten. Die Anwendung von Phytotherapeutika ist für Verbrennungen Grad I und II geeignet.

\section{Stationäre Aufnahme}

Bei einer Verbrennungsfläche von mehr als $10-15 \%$, bei Kindern 5-10\%, Verbrennungen im Gesichts-, Hand/Fußbereich, GenitoAnal-Bereich, Respirationstrakt, Verbrennungen durch Strom oder nicht einzuschätzenden Intoxikationen ist eine stationäre Aufnahme des Patienten anzuraten. Es empfiehlt sich hier zum Transport das Abdecken der Wunden z. B. mit Metalline ${ }^{\circledR}$ Wundauflagen oder Alufolie. Neben der Wundbehandlung sind eine Volumensubstitution inkl. Flüssigkeitsbilanzierung und eine Schmerzbehandlung unabdingbar (Tab. 1).

Bei Verbrennungen III. und IV. Grades sind chirurgische Interventionen meist erforderlich (Nekrektomie, Débridement, Transplantation mit Spalthauttransplantaten, Meshgraft-Transplantaten, Allooder Xenotransplantate). Spezialkliniken (sog. Verbrennungszentren) sind dem Rettungsdienst bekannt und können lebensrettend bei stark ausgeprägten Verbrennungen sein.

Merke: Grundsätzlich an Tetanusprophylaxe, Volumensubstitution sowie Schmerzmedikation denken.

\section{Heilungsprognosen bei Verbrennungen}

- I. Grades: Nach wenigen Tagen Abheilung

- II. Grades: Restitutio ad integrum nach 2-4 Wochen.

- III./IV. Grades: Narbige Abheilung. Cave! Keloidbildung.

Etablierte und neue Phytotherapeutika [2-10] Echte Aloe (Aloe vera)

Der Blätterextrakt aus der Aloe-vera-Pflanze wirkt antientzündlich und proliferationsfördernd. 
Anwendung: Das Auftragen der Pflanze wird in manchen Ländern seit Jahrhunderten als optimales Mittel zur Therapie von Verbrennungen Grad I-II angewandt. Alkoholfreie Gele oder hydratisierende Lotionen als Fertigarzneimittel können hochprozentig eingesetzt werden.

Unerwünschte Wirkungen: nicht bekannt.

\section{Ananas (Ananas comosus)}

Bromelain ist das Protein, das für die entzündungs- und ödemhemmende Wirkung der Ananas verantwortlich ist.

Anwendung: Tagesdosis von 80-240 mg Bromelain in 2-3 Einzeldosen als Tabl./Kaps.

Unerwünschte Wirkungen: Magenbeschwerden, Diarrhöen, selten allergische Reaktionen.

\section{Birkenrinde (Betula alba)}

Hier zeigen in den letzten Jahren neuere Studien eine gute antiinflammatorische Wirksamkeit.

Anwendung: Fertigarzneimittel stehen für Verbrennungen auch in Kombination mit Harnstoff zur Steigerung der Hydration zur Verfügung.

Unerwünschte Wirkungen: nicht bekannt.

Eichenrinde (Cortex quercus)

Vorwiegend adstringierende Wirkung.

Anwendung: Feuchte Umschläge mit Wurzelextrakt (20g feingeschnittene Wurzel in einem Liter Wasser aufkochen, 5-10 min ziehen lassen, abseihen - abkühlen. Alkoholhaltige Fertigarzneimittel für Umschläge sind bei ausgeprägten Verbrennungen nicht empfehlenswert.

Unerwünschte Wirkungen: Aufgrund der adstringierenden Wirkung kann ein Spannungsgefühl entstehen, evtl. Rückfettung mit hydratisierenden Lotionen.

\section{Grüner Tee (Camellia sinensis)}

Hohe Anteile von Poliphenolen und den Inhaltsstoffen Catechin, Epicatechin und Epigallocatechin fördern die Wundheilung und wirken antiinflammatorisch. Es wurde gezeigt, dass sowohl die lokale Anwendung als auch durch Trinken von grünem Tee präventiv UV-induzierter Inflammation vorgebeugt werden kann.

Anwendung: Lokal: Grünen Tee aufbrühen - wie in der Gebrauchsanweisung empfohlen, abkühlen lassen und mit feuchten Umschlägen auftragen, 10-15 min, danach Umschlag erneuern.

Unerwünschte Wirkungen: ggf. austrocknend - Rückfettung erforderlich.

\section{Kamille (Flores Chamomilla)}

Wundheilungsfördernd, antibakteriell.

Anwendung: 3-10\% Kamillenblätter als Umschläge oder in Lotionen oder Cremes bei subakuten Entzündungen.

Unerwünschte Wirkungen: Allergien sind beschrieben.

\section{Reseda luteola L. (Färber-Wau)}

Das Flavonoid Luteolin ist der wirksamste Inhaltsstoff dieser Pflanze. Luteolin wirkt antiinflammatorisch, antioxidativ und photoprotektiv. Kombinationen mit Tocopherol und Ubiquinone erhöhen die photoprotektiven Wirkungen.

Unerwünschte Wirkungen: nicht bekannt.

\section{Ringelblume (Calendula officinalis)}

Adstringierende, antiphlogistische, antiödematöse Wirkung (s. auch Heilpflanzenporträt S. 44).

Anwendung: 1-2g der Blüten/Blätter mit $150 \mathrm{ml}$ Wasser übergießen 7-10 min ziehen lassen, absehen, abkühlen lassen. Fertigarzneimittel in wenig fettender aber hydratisierender Grundlage können angewendet werden.

Unerwünschte Wirkungen: Allergien sind beschrieben.

\section{Sonnenhut (Echinacea purpurea)}

Echinacea und das darin enthaltende Echinacosid hat antiinflammatorische und wundheilungsfördernde Eigenschaften. Sonnenhut ist für seine Wirksamkeit zur unterstützenden Therapie bei oberflächlichen Wunden bekannt.

Anwendung: Lokal in hydratisierenden Grundlagen.

Unerwünschte Wirkungen: Allergien sind beschrieben, vorwiegend bei Patienten mit Allergien auf Korbblütler.

\section{Weihrauch (Boswellia serrata, Boswellia)}

Bei verschiedenen Boswellia-Arten wurden antiinflammatorische Eigenschaften gezeigt. Der Gehalt der Triterpene aus dem Boswelliaharz variiert stark von Spezies zu Spezies und ist abhängig von Erntezeitpunkt.

Anwendung: Arzneimittel zur oralen und lokalen Anwendung sind auf dem Markt aus unterschiedlichen Stammpflanzen.

Unerwünschte Wirkungen: bislang nicht bekannt

\section{Zaubernuss (Hamamelis) - Rinde und Blätter}

Vorwiegend adstringierende Wirkung.

Anwendung: Tees oder feuchte Umschläge mit Extrakt (5-10g Hamamelisrinde- oder Blätter mit einer Tasse kochendes Wasser übergießen, 10 min ziehen lassen - anschließend abseihen), als Tee trinken oder/und als feuchte Umschläge anwenden. Fertigarzneimittel mit Extrakten, Cremes, Salben und Lotionen sind bekannt. Bei Verbrennungen sollten feuchte Umschläge ohne Alkohol und halbfeste Zubereitungen wie Lotionen vorwiegend aufgrund der Hydratisierungspotenz zur Anwendung kommen.

Unerwünschte Wirkungen: ggf. austrocknend - Rückfettung erforderlich.

\section{Sonnenschutz allgemein}

Im 21. Jahrhundert ist bekannt, dass zu intensives Sonnenbaden das Auftreten von Hauttumoren forciert und die Hautalterung beschleunigt. In einer amerikanischen Studie mit 18 Billionen Patientenvisiten wurden Ärzte (Dermatologen, Allgemeinmediziner, Kinderärzte) befragt, wie oft sie über Sonnenschutz aufklären. 0,1\% gaben an über Sonnenschutz allgemein aufzuklären; bei diagnostizierten Hauterkrankungen waren es $1 \%$. Am häufigsten wurden Patienten über 80 Jahre (vorwiegend bei Lichtschäden), am wenigsten Kinder über die Gefahren und Risiken einer intensiven Sonnenaussetzung und die Möglichkeiten des Sonnenschutzes aufgeklärt [1].

Grundsätzlich sollte immer ausreichender Sonnenschutz in Erwägung gezogen werden. Präventiv sind bestimmte Regeln beim Sonnenbaden zu beachten, s. Tab. 2. Sonnenschutzpräparate mit ausreichendem UVA- und UVB-Schutz sind in vorwiegend hydratisierender Grundlage, z.B. Milch, empfehlenswert. Die Höhe des Lichtschutzfaktors ist vom Hauttyp, der Jahreszeit, dem Ausmaß und dem Ort des Sonnendbadens abhängig. 


\begin{tabular}{|c|c|}
\hline \multicolumn{2}{|c|}{ Regeln für gesundes Sonnenbaden } \\
\hline Regel & Umsetzung \\
\hline $\begin{array}{l}\text { Mittagssonne } \\
\text { meiden }\end{array}$ & $\begin{array}{l}\text { Keine Sonne zwischen } 11 \text { und } 15 \text { Uhr, } \\
\text { hier ist die UV-Strahlung am stärksten. }\end{array}$ \\
\hline Schatten & $\begin{array}{l}\text { Zeit nehmen, sich an die Sonne zu } \\
\text { gewöhnen. Die ersten Urlaubs- und } \\
\text { Sommertage den Schatten bevor- } \\
\text { zugen. }\end{array}$ \\
\hline Kleidung \& Cap & $\begin{array}{l}\text { Dichte, lockere Kleidung tragen und } \\
\text { auf jeden Fall eine Kopfbedeckung. }\end{array}$ \\
\hline T-Shirt beim Baden & $\begin{array}{l}\text { Schutz beim Baden mit T-Shirt und } \\
\text { wasserfester Sonnencreme. Und } \\
\text { nach dem Baden Nachcremen nicht } \\
\text { vergessen! }\end{array}$ \\
\hline $\begin{array}{l}\text { Sonnencreme > } \\
\text { LSF } 20\end{array}$ & $\begin{array}{l}\text { Eine halbe Stunde vor dem Sonnen- } \\
\text { bad eincremen, damit sie die Wirkung } \\
\text { voll entfalten kann. }\end{array}$ \\
\hline Nachcremen & $\begin{array}{l}\text { Den Sonnenschutz mehrmals täglich } \\
\text { erneuern. Nachcremen verlängert } \\
\text { nicht die Schutzwirkung. }\end{array}$ \\
\hline 50 Sonnenbäder & $\begin{array}{l}\text { Nicht mehr als } 50 \text { Sonnenbäder im } \\
\text { Jahr. Dazu zählt jeder Aufenthalt in } \\
\text { Sonne und Solarien! }\end{array}$ \\
\hline NEIN zu Solarien & $\begin{array}{l}\text { Meide Solarien! Die UV-Intensität ist } \\
\text { hier besonders stark und schadet } \\
\text { empfindlicher Haut. }\end{array}$ \\
\hline
\end{tabular}

Tab. 2 Quelle adaptiert: Arbeitsgemeinschaft Dermatologische Prävention. http://www.derma.de

Interessenkonflikt: Die Autorin erklärt, dass keine wirtschaftlichen oder persönlichen Verbindungen bestehen oder bestanden haben.

\section{Online zu finden unter}

http://dx.doi.org/10.1055/s-0034-1390666

\section{Literatur}

1 Akamine K et al. Trends in Sunscreen Recommendation Among US Physicians. JAMA Dermatology 2014; 150: 51-55

$\overline{2}$ Augustin M, Hoch Y. Phytotherapie bei Hauterkrankungen. Grundlage Praxis - Studien. München: Elsevier; 2004

3 Casetti F, Wölfle U, Gehring W, Schempp CM. Dermocosmetics for Dry Skin: A New Role for Botanical Extracts. Skin Pharmakol Physiol 2011; 24 (6): 289293

$\overline{4}$ Kraft K. Phytotherapie. In: Kraft K, Stange R. Naturheilverfahren. Stuttgart: Hippokrates; 2010: 145-180

5 Mostafa DM, Ammar NM, Basha M, Hussein RA, El Awdan S, Awad G. Transdermal microemulsions of Boswellia carterii Bird: formulation, characterization and in vivo evaluation of anti-inflammatory activity. Drug Deliv 2014 Apr 14. [Epub ahead of print]

6 Pazygar N, Yaghoobi R, Rafiee E, Mehrabian A, Feily A. Skin Wound Healing and Phytomedicine. A Review. Skin Pharmacol Physiol 2014; 27: 303-310

7 Reuter J, Merfort I, Schempp C. Botanicals in Dermatology. Am J Clin Dermatol 2010; 11: 247-267
8 Reuter J, Wölfle U, Weckesser St, Schempp C. Which plant for which skin disease? Part 1: atopic dermatitis, psoriasis, acne, condyloma and herpes simplex. JDDG 2010; 10: 788-796

$\overline{9}$ Reuter J, Wölfle U, Korting C, Schempp C. Which plant for which skin disease? Part 2: Dermatophytes, chronic venous insufficiency, photoprotection, actinic keratosis, vitiligo, hair loss, cosmetic indications. JDDG 2010; 11: 866-873

$\overline{10}$ Wölfle U, Haarhaus B, Schempp CM. The photoprotective and antioxidative properties of luteolin are synergistically augmented by tocopherol and ubiquinone. Planta Med. 2013 Jul;79(11): 963-5.

$\overline{11}$ Wollina U. Combustio und Ambustio. In: Plewig, Landthaler, Burgdorf, Hertl, Ruzicka. Braun-Falco's Dermatologie, Venerologie und Allergologie. 6. Aufl. Heidelberg: Springer; 2012: 733-735

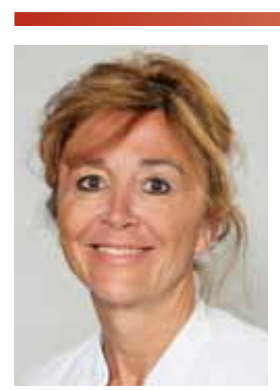

Priv. Doz. Dr. Petra Staubach

Hautklinik und Poliklinik

Universitätsmedizin Mainz

Langenbeckstraße 1

55131 Mainz

petra.staubach@unimedizin-mainz.de

Petra Staubach ist als Dermatologin und Allergologin in der Universitätsklinik Mainz als Oberärztin tätig. Sie leitet das klinische Studienzentrum und verschiedene Spezialsprechstunden. Schwerpunkte: Lokaltherapien inkl. Rezepturen, Naturheilverfahren, Ernährungsmedizin, Psychosomatik und Qualitätsmanagement. Sie ist im Vorstand des deutschen UrtikariaNetzwerks (www.urtikaria.net) und der Gesellschaft für Dermopharmazie e. V. 